\title{
The Combination of Pairwise and Group Interactions Promotes Consensus in Opinion Dynamics
}

\author{
Xiaoxuan Liu, ${ }^{1}$ Changwei Huang $\mathbb{D}^{2,3}$ Haihong Li, ${ }^{1}$ Qionglin Dai $\mathbb{D}^{1},{ }^{1}$ and Junzhong Yang ${ }^{1}$ \\ ${ }^{1}$ School of Science, Beijing University of Posts and Telecommunications, Beijing 100876, China \\ ${ }^{2}$ School of Computer, Electronics and Information, Guangxi University, Nanning 530004, China \\ ${ }^{3}$ Guangxi Key Laboratory of Multimedia Communications and Network Technology, Guangxi University, \\ Nanning 530004, China \\ Correspondence should be addressed to Changwei Huang; cwhuang@gxu.edu.cn and Qionglin Dai; qldai@bupt.edu.cn
}

Received 26 July 2020; Revised 23 October 2020; Accepted 15 January 2021; Published 27 January 2021

Academic Editor: Wei Zhou

Copyright ( 2021 Xiaoxuan Liu et al. This is an open access article distributed under the Creative Commons Attribution License, which permits unrestricted use, distribution, and reproduction in any medium, provided the original work is properly cited.

\begin{abstract}
In complex systems, agents often interact with others in two distinct types of interactions, pairwise interaction and group interaction. The Deffuant-Weisbuch model adopting pairwise interaction and the Hegselmann-Krause model adopting group interaction are the two most widely studied opinion dynamics. In this study, we propose a novel opinion dynamics by combining pairwise and group interactions for agents and study the effects of the combination on consensus in the population. In the model, we introduce a parameter $\alpha$ to control the weights of the two interactions in the dynamics. Through numerical simulations, we find that there exists an optimal $\alpha$, which can lead to a highest probability of complete consensus and minimum critical bounded confidence for the formation of consensus. Furthermore, we show the effects of $\alpha$ on opinion formation by presenting the observations for opinion clusters. Moreover, we check the robustness of the results on different network structures and find the promotion of opinion consensus by $\alpha$ not limited to a complete graph.
\end{abstract}

\section{Introduction}

Social behaviors are shaped by opinions and beliefs. In the real world, people hold opinions in various aspects of social life from the judgments for goods in daily life to the evaluation of candidates in elections. By interacting with other ones, people may change their opinions. The evolution of opinions could significantly affect the social life, including the formation of culture, language, mainstream views, social norms, and so on. Therefore, the study of opinions dynamics has attracted much attention of researchers from a wide variety of fields, from statistical physics to sociology to communication science [1-6].

According to whether the opinions take discrete values or not, the opinion dynamics can be divided into two categories, discrete opinion dynamics and continuous opinion dynamics. Typical discrete opinion dynamics include the Galam majority-rule model [5], the voter model [7], the naming game [8], the Sznajd model [9], and the social impact model [10]. Meanwhile, the Deffuant-Weisbuch (DW) model [11] and Hegselmann-Krause (HK) model [12] are the representative ones of continuous opinion dynamics.

In the original DW model with pairwise interaction, there are two key parameters, the bounded confidence $\varepsilon$ in the range of $[0,1]$ and the convergence rate $\mu$ in the range of $[0,0.5]$. In an elementary evolution step, two neighboring individuals $(i, j)$ are chosen randomly. If the difference of their opinions is less than the bounded confidence $\varepsilon$, they will update their own opinions $x_{i}(t)$ and $x_{j}(t)$ with $x_{i}(t+$ $1)=x_{i}(t)+\mu\left[x_{j}(t)-x_{i}(t)\right]$ and $x_{j}(t+1)=x_{j}(t)+\mu\left[x_{i}(t)\right.$ $\left.-x_{j}(t)\right]$, respectively. The convergence rate $\mu$ can be used to describe how fast they are shifted towards each other. In the early work by Fortunato [13], the author found that the threshold value of $\varepsilon$ above which all agents share the same opinion in the final configuration (consensus) is 0.5 in the DW model, independently of the underlying social topology. In [14], the authors modified the DW model by introducing heterogeneous convergence rate and found that the decrease 
of the convergence rate leads to the decrease of the confidence threshold. Furthermore, considering that individuals may form different network structures in the real world, Shang [15] studied the evolution of opinions by using the DW model on multiplex networks and found that the existence of multiplexity restrains the convergence. In [16], Chen et al. studied the DW model on a ring with repelling mechanism and circular opinions. Their results showed that the interplay between the repelling mechanism and the circular opinion may lead to a time-dependent opinion dynamics. Moreover, opinion dynamics based on the DW model have also been investigated on evolving and adaptive networks [17]. The authors obtained a counterintuitive finding that the diversity of opinions facilitates consensus formation.

In contrast, in an elementary evolution step in the HK model, each agent updates his/her opinion $x_{i}(t)$ by taking into account all of the neighbors as long as their opinion differences are within the bounded confidence. Thus, the opinion updating is implemented through group interaction in the HK model, which is fundamentally different from the pairwise interaction in the DW model. Generally, the agent $i$ updates opinion by taking the average of his/her neighbors' opinions including his/her own $[12,18]$. In the traditional HK model, the bounded confidence is the only parameter. Many previous studies on the HK model have considered the effects of heterogeneous bounded confidence on consensus [19-22]. Lorenz [19] found that, in a society with two different bounded confidences, consensus could be reached even when bounded confidences are significantly below the critical bounded confidence in a homogeneous society. In [20], the agents are divided into three categories, each with a different bounded confidence interval. The authors studied how agents of each category and the population size affect opinion dynamics. In addition, one of personal characteristics, the so-called stubbornness, has also been considered in the HK model. Stubbornness depicts that an agent adheres to his/her own opinion to some extent and can affect the opinion dynamics significantly [23-28]. In [23], the authors introduced a parameter characterizing the extent to which an agent trust himself/herself and considered heterogeneous stubbornness. In their model, they used the HK model to present a public opinion control strategy based on public authority. Furthermore, in [24], Han et al. investigated a modified HK model with heterogeneous bounded confidences and stubbornness. Besides, some new perspectives have also been considered in the study of opinion dynamics based on the HK model. For example, Shang and Chen [29] proposed an opinion dynamics in which each agent can adjust his/her opinion by using an incremental proportional-integral controller and found that the strong integral effect can promote the system to achieve consensus easily and reduce the number of subsets fast.

In the above works, the researchers focused on only one of the two models, the DW model and the HK model. However, sometimes the combination of the characteristics of the two models might be more realistic. Consider that, in the real world, people are simultaneously influenced by their circumstances from different aspects. Some impacts might come from a small environment, for instance, from the partner or family, while others may come from general social environments, such as company and school. From an opinion dynamics perspective, these two types of impacts on opinion could be well described by the interactions adopted in the DW model and the HK model, respectively. The former one is the type of pairwise interaction, corresponding to the situation of a small environment, while the latter one is group-based, fitting for the case of a general environment. Generally, the pairwise and group interactions work together to shape one's ideas or opinions in real life. Therefore, we are interested to study the opinion dynamics in which both types of interactions are involved in. Furthermore, from an engineering point of view, there would be a cost for interactions between/ among individuals which are realized by data transmission and information exchange. Obviously, in the DW model and the HK model, different types of interactions need different communication costs. Thus, we wonder that whether an optimization of the condition for consensus could be found when these two models are combined. If the answer is yes, this method might be also applied to engineering fields to save communication resources and reduce computational complexity. Besides, the idea of combining different characteristics in the opinion dynamics has been considered in previous studies. For example, Chen et al. [30] considered two cognitive styles, field-dependence and field-independence, in public opinion dynamics and proposed a public opinion evolution model based on cognitive styles (CS model). In their model, the individual combines the opinions of neighbors and experiences of himself/herself to update his/her own opinion. Different from their work, we combine two different interactions described by the DW and the HK models, instead of two cognitive styles of individuals. Particularly, we introduce a parameter to control the weights of the two types of interactions and focus on how the combination affects the consensus of the population.

The rest of this study is organized as follows. In Section 2, we introduce the model of the opinion dynamics in detail. In Section 3, we present the results and discussion. Finally, we give a brief summary in Section 4 .

\section{Model}

Consider a population with $N$ agents who are located at a complete graph or certain complex network. We set $N=$ 1000 throughout this work. Each agent $i$ holds an opinion $x_{i}(t)$, which ranges from 0 to 1 and evolves with time $t$. Initially, each agent is randomly assigned with an opinion, and the opinions in the population are uniformly distributed. In each elementary evolution step, a pair of neighboring agents $(i, j)$ is randomly chosen. If the network is a complete graph, each agent has $N-1$ neighbors. Otherwise, the neighboring agents are determined by the underlying complex networks. Generally, it is assumed that each agent only considers interacting with their neighbors whose opinions differ from his/her own within a certain confidence level, so-called the bounded confidence. For simplicity, we adopt homogeneous bounded confidence $\varepsilon$ for the whole population. 
The opinion updates include two ingredients. The first ingredient is determined by the pairwise interaction based on the DW model. We check whether $\left|x_{i}(t)-x_{j}(t)\right|<\varepsilon$. If it is true, the two individuals update their opinions based on a compromise strategy. That is, after a constructive debate, the two interacting individuals get closer to each other. Otherwise, nothing happens. This ingredient can be formulated as

$$
\begin{aligned}
& x_{i}^{\prime}(t+1)= \begin{cases}x_{i}(t)+\mu\left[x_{j}(t)-x_{i}(t)\right], & \left|x_{i}(t)-x_{j}(t)\right|<\varepsilon, \\
x_{i}(t), & \text { otherwise, }\end{cases} \\
& x_{j}^{\prime}(t+1)= \begin{cases}x_{j}(t)+\mu\left[x_{i}(t)-x_{j}(t)\right], & \left|x_{j}(t)-x_{i}(t)\right|<\varepsilon, \\
x_{j}(t), & \text { otherwise. }\end{cases}
\end{aligned}
$$

The convergence rate $\mu$ characterizes how fast the agents are willing to update their opinions towards each other in one round. Here, we set $\mu$ to be 0.5 , which means that the two individuals will converge to their average opinions.

The second ingredient is based on the HK model. Following the traditional HK model, the evolution of agents' opinions can be defined as

$$
\begin{aligned}
& x_{i}^{\prime \prime}(t+1)=\frac{1}{\left\|N_{i}(t)\right\|} \sum_{k \in N_{i}(t)} x_{k}(t), \\
& x_{j}^{\prime \prime}(t+1)=\frac{1}{\left\|N_{j}(t)\right\|} \sum_{k \in N_{j}(t)} x_{k}(t),
\end{aligned}
$$

where $N_{i}(t)$ is the set of agent $i$ 's neighbors (including agent i) whose opinions satisfy $\left|x_{i}(t)-x_{k}(t)\right|<\varepsilon$ at time $t$, and $\left\|N_{i}(t)\right\|$ is the number of the agents in the set $N_{i}(t)$.

In this work, we combine above two ingredients and assume that the agents update their opinions according to the equations given by

$$
\left\{\begin{array}{l}
x_{i}(t+1)=(1-\alpha) x_{i}^{\prime}(t+1)+\alpha x_{i}^{\prime \prime}(t+1), \\
x_{j}(t+1)=(1-\alpha) x_{j}^{\prime}(t+1)+\alpha x_{j}^{\prime \prime}(t+1),
\end{array}\right.
$$

in which $\alpha$ is a tunable parameter controlling the weights of the two ingredients. If $\alpha=0$, the model degenerates to the original DW model. At the other extreme $\alpha=1$, the model turns into the original HK model.

In order to ensure that each agent has a chance to interact with his/her neighbor once on average, we take $N / 2$ elementary steps described above in one full Monte Carlo step (MCS). In each MCS, we monitor the opinion changes between two successive MCSs for all the agents whose opinions are updated. If the opinion changes are less than $10^{-10}$ for all the agents in successive 50 MCSs, we claim that a steady state is reached.

\section{The Numerical Results and Discussion}

We start from the model on a complete graph. We first consider the probability of complete consensus $P_{c}$. We simulate the model for $2000 \sim 5000$ different realizations. $P_{c}$ is defined as the fraction of the realizations in which consensus is reached. The probability of complete consensus $P_{c}$ as a function of the bounded confidence $\varepsilon$ at several different $\alpha$ is shown in Figure 1. When $\varepsilon$ is small, the system can not reach consensus and $P_{c}$ is equal to 0 . With $\varepsilon$ increasing, $P_{c}$ starts to be nonzero. It has been found that, for the original DW model, the critical bounded confidence is at $\varepsilon=0.5$ for the complete consensus. However, $\varepsilon=0.5$ is the threshold for $P_{c}=1$, and nonzero $P_{c}$ actually appears at around $\varepsilon=0.25$, a value for the formation of polar state in which population breaks down into two noninteracting subpopulations. Between $\varepsilon=0.25$ and 0.5 , there exists a window of $\varepsilon$ for nonzero $P_{c}$ [14]. Such a window with nonzero $P_{c}$ can be observed in our model. We denote the critical value of $\varepsilon$ which leads to a nonzero $P_{c}$ by $\varepsilon_{c 1}$. As shown in Figure 1, for $\alpha=0$, when the opinion dynamics only contain the pairwise interactions described by the DW model, $\varepsilon_{c 1}=0.25$, and the window of nonzero $P_{c}$ exists for $\varepsilon \in(0.25,0.35)$, which is consistent with the result in the previous study [14]. One can find that $\varepsilon_{c 1}$ always decreases with the increase of $\alpha$. On the other hand, small $\alpha$ leads to the expanse of the window of nonzero $P_{c}$. For intermediate $\alpha(\alpha=0.1)$, the window of nonzero $P_{c}$ disappears and $P_{c}$ rises all the time till it reaches 1 . For large $\alpha$, a rapid increase of $P_{c}$ could be observed when $\varepsilon$ exceeds $\varepsilon_{c 1}$, while followed by a slightly decrease and then rises again. Finally, when $\varepsilon$ is large enough, $P_{c}$ goes to 1 for all $\alpha$. Either the less $\varepsilon_{c 1}$ or the expansion of the window of nonzero $P_{c}$ with $\alpha$ suggests that the inclusion of the group interaction favors the consensus in the population. Furthermore, it could be found that $P_{c}$ reaches 1 when $\varepsilon$ is above another critical value. We denote this critical value of $\varepsilon$ by $\varepsilon_{c 2}$. These critical values for different $\alpha$ are marked by the arrows of corresponding color shown in Figure 1, which will be discussed in more detail later.

The formation of window of $\varepsilon$ for nonzero $P_{c}$ can be understood by the bridge effect proposed in [14]. The bridge effect is explained as follows. Consider two opinion clusters with a distance larger than $\varepsilon$. The two opinion clusters cannot interact with each other directly. However, if there exist certain agents holding opinions in between and the opinion differences between these agents and the opinion clusters less than $\varepsilon$, these two opinion clusters may indirectly interact with each other under the help of these agents. For the original DW model $(\alpha=0)$, the states with $n$ opinion clusters is determined by the condition $n=1 / 2 \varepsilon$. The state with three opinion clusters transitions to the polarization state with two opinion clusters at $\varepsilon=0.25$. When $\varepsilon$ is just little larger than 0.25 , the transition to the polarization state requires a long transient. During the transient time, there may exist agents with such opinions that they are possible to be recruited by the two opinion clusters. The long transient enhances the indirect interaction between the two clusters, which makes these two clusters merge into a consensus state. However, when $\varepsilon$ is not close to 0.25 , the transient time is so short that the indirect interaction is weak for the two opinion clusters, and the consensus state is hard to be formed. Consequently, we observe a narrow window of nonzero $P_{c}$ at $\varepsilon$ close to 0.25 for $\alpha=0$ in Figure 1. When $\alpha$ increases from zero, the group interaction described by the $\mathrm{HK}$ model steps in. In the presence of the HK group 


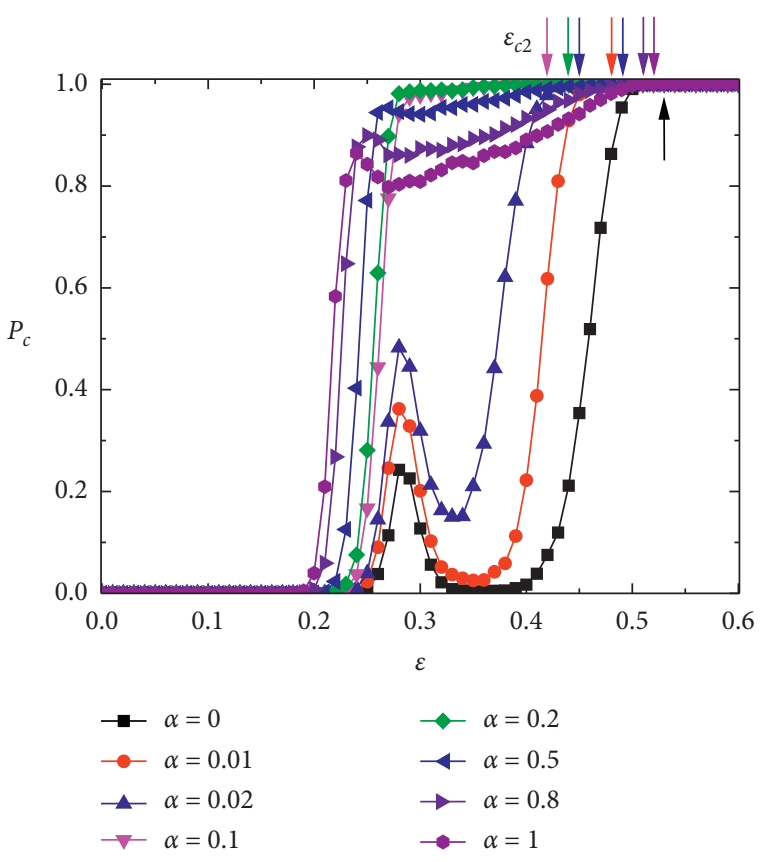

FIGURE 1: The probability of complete consensus $P_{c}$ as a function of $\varepsilon$ at $\alpha=0,0.01,0.02,0.1,0.2,0.5,0.8$, and 1 . The arrows of corresponding color indicate the critical values of $\varepsilon$, denoted by $\varepsilon_{c 2}$, at which $P_{c}=1$ can be obtained for different $\alpha$.

interaction, if there exist agents with opinions in between two opinion clusters, these opinions may stay in between for a long time and the interaction between the two clusters may be assisted by the agents holding these opinions. Such an effect becomes stronger with the increase of $\alpha$, which is in agreement with the observation that the window of nonzero $P_{c}$ is enlarged with $\alpha$. On the other hand, for the original HK model $(\alpha=1), P_{c}$ increases from zero when $\varepsilon>0.2$, which covers the window of nonzero $P_{c}$. Therefore, when $\alpha$ becomes sufficiently large, the nonmonotonic of $P_{c}$ with $\varepsilon$ due to the window of $\varepsilon$ for nonzero $P_{c}$ is replaced by a monotonic increase.

Figure 1 shows that, for intermediate values of $\varepsilon$, there exists an optimal $\alpha$, which leads to a maximum $P_{c}$. In order to present a clear picture for the optimization of $P_{c}$ with $\alpha$, we show the probability of complete consensus $P_{c}$ against $\alpha$ at several different $\varepsilon$ in Figure 2. From Figure 2, one can see that, when $\alpha$ is small, $P_{c}$ increases rapidly with the increase of $\alpha$ and reaches a quite high level, even close or equal to 1 . In a certain range of $\alpha$ (from about 0.1 to 0.3 ), $P_{c}$ can hold a high value. Then, with the further increase of $\alpha, P_{c}$ decreases slowly. It can remain in a relatively high level greater than 0.8 , even when $\alpha=1$. The results are consistent with those shown in Figure 1. In addition, although the differences between these curves for different $\varepsilon$ are not great, we still can find that, the larger the bounded confidence $\varepsilon$ is, the higher consensus probability $P_{c}$ can be obtained. The optimization of $P_{c}$ by $\alpha$ can also be explained by the bridge effect. For small $\alpha$, the pairwise interaction due to the DW model is dominant. The formation of distant two opinion clusters is due to the pairwise interaction. However, the opinions between two clusters may stay due to the group interaction

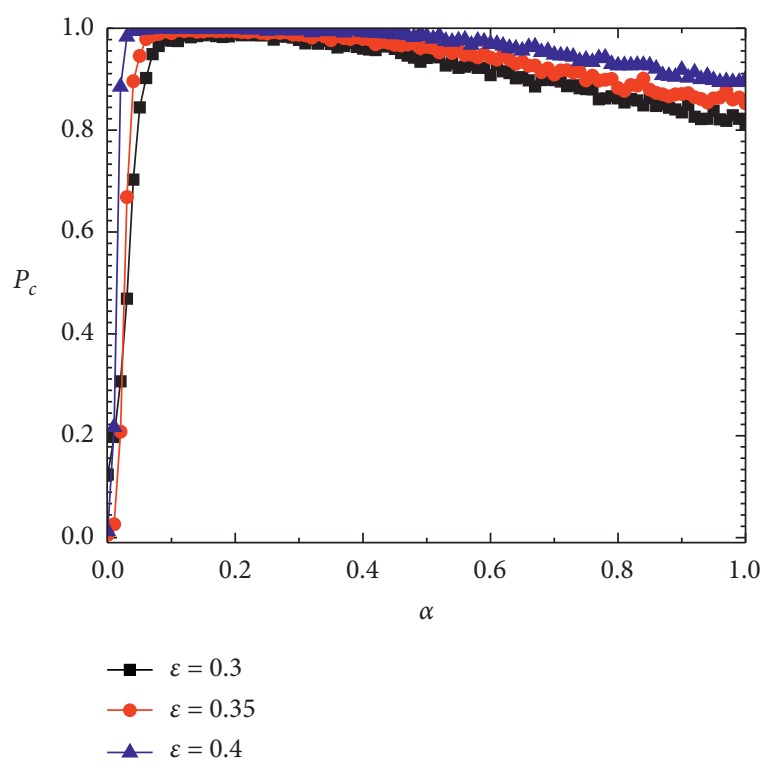

FIgURE 2: The probability of complete consensus $P_{c}$ as a function of $\alpha$ at $\varepsilon=0.3,0.35$, and 0.4 . The optimization of $P_{c}$ by $\alpha$ could be obviously found.

of the HK model, which leads to the increase of $P_{c}$. The bridge effect increases with $\alpha$. On the other hand, for large $\alpha$, the group interaction due to the HK model is dominant, and the distant two opinion clusters forms due to the group interaction. However, in simulations, we find that the transient time in the HK model is greatly longer than that in the DW model. In the formation of two clusters due to the group interaction, the pairwise interaction makes some agents with opinions hard to be recruited by these clusters. These agents play the roles of producing bridge effects and assist the merging of opinion clusters into one. This type of bridge effect due to the pairwise interaction gets enhanced with the decrease of $\alpha$. Based on the above two types of bridge effects, the optimization of $P_{c} \alpha$ appears. Actually, the optimization of $P_{c}$ with $\alpha$ reflects the collective contribution of the pairwise interaction and the group interaction to the opinion dynamics.

In correspondence with the optimization of $P_{c}$ by $\alpha$, the critical value $\varepsilon_{c 2}$ at which $P_{c}=1$ could be obtained also displays an optimization by $\alpha$. To show it, we present $\varepsilon_{c 2}$ as a function of the parameter $\alpha$ in Figure 3. For small $\alpha, \varepsilon_{c 2}$ decreases rapidly with the increase of $\alpha$ and reaches its minimum approximately at $\alpha=0.1$. Then, $\varepsilon_{c 2}$ rises again with the increase of $\alpha$. The results are consistent with those shown in Figure 1. It can be concluded that the combination of the pairwise and group interactions can effectively reduce $\varepsilon_{c 2}$, since it supports wider parameter regions for the system to reach the complete consensus.

During the evolution process, opinion clusters can be formed through the interactions among agents. Generally speaking, in the beginning stage, small opinion clusters are formed, and then, small opinion clusters expand. The relatively large opinion clusters may invade smaller ones. Finally, the population tends to be consensus or split into two (polarization) or more (fragmentation) clusters with the 


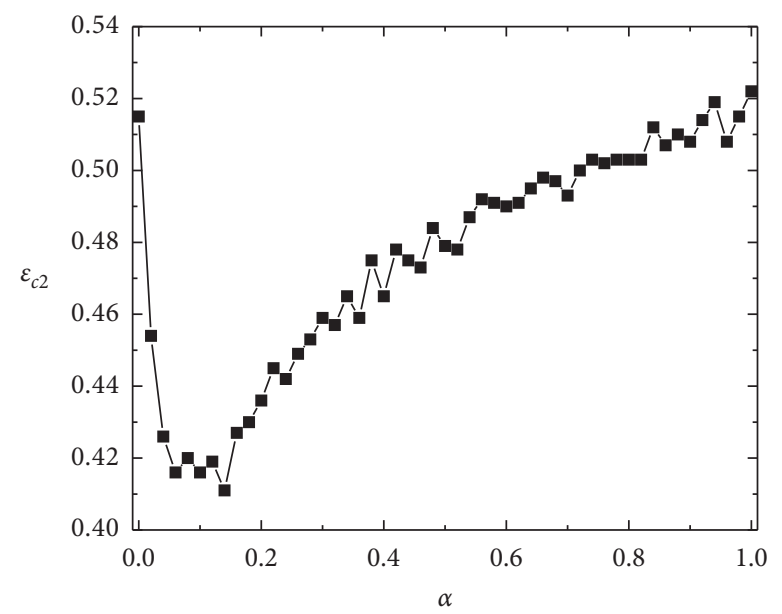

Figure 3: The critical bounded confidence $\varepsilon_{c 2}$, over which the system can always reach complete consensus, as a function of $\alpha$.

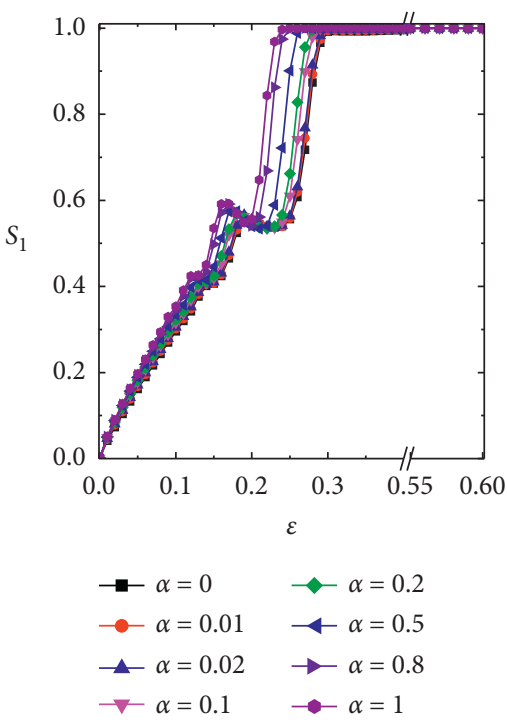

(a)

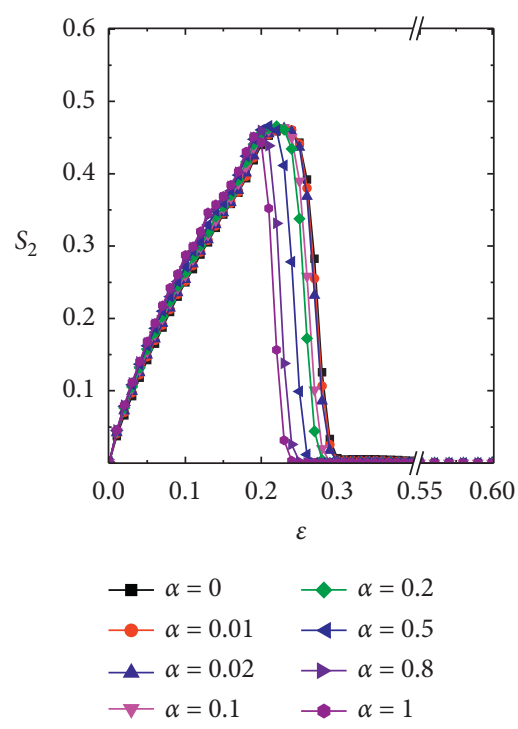

(b)

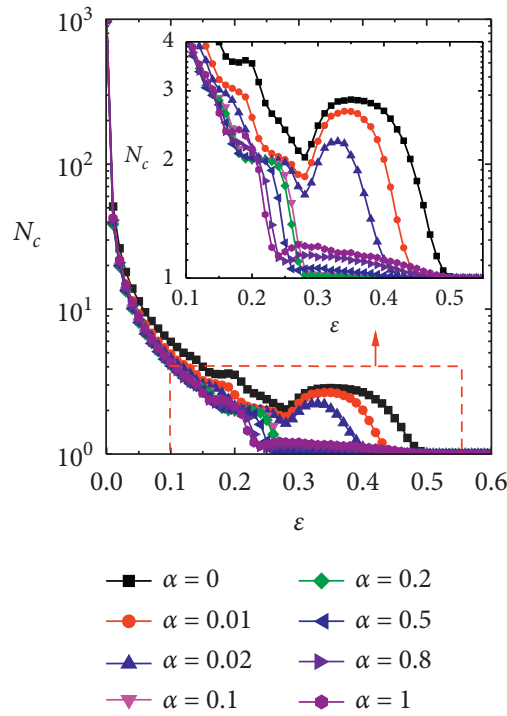

(c)

Figure 4: (a) The relative size of the largest opinion cluster $S_{1}$ as a function of $\varepsilon$ at different $\alpha$. (b) The relative size of the second largest opinion cluster $S_{2}$ as a function of $\varepsilon$ at different $\alpha$. (c) The number of clusters $N_{c}$ as a function of $\varepsilon$ at different $\alpha$. An enlargement of the region for small $N_{c}$ is shown in the inset. $\alpha=0,0.01,0.02,0.1,0.2,0.5,0.8$, and 1 .

same opinion in each of them. Thus, large opinion clusters play a critical role in the final opinion formation. Now, we are interested to explore the impact of $\varepsilon$ on the relative sizes of the largest and the second largest opinion clusters (denoted by $S_{1}$ and $S_{2}$, respectively) and the number of clusters $N_{c}$. To measure the cluster size and count the number of clusters in the steady state of the opinion dynamics, we need to define opinion clusters. To do it, we sort out agents in the ascending order of their opinions. Two successive agents belong to different opinion clusters if the opinion difference between them is larger than the bounded confidence. The results are shown in Figure 4. When $\varepsilon$ is small $(\varepsilon<0.2), S_{1}$ and $S_{2}$ both increase with the increase of $\varepsilon$ in a similar way. The system evolves into many opinion clusters (shown by $N_{c}$ in Figure 4(c)). The population cannot reach the state of complete consensus. When $\varepsilon$ further increases, $S_{1}$ increases while $S_{2}$ decreases. At the same time, the number of clusters $N_{c}$ decreases rapidly. When $\varepsilon$ is close to $\varepsilon_{c 1}, S_{1}$ approximately equals to 1 , which means that most of the agents form a giant opinion cluster. Meanwhile, $S_{2}$ drops close to 0 , and $N_{c}$ becomes very small. Finally, when $\varepsilon$ is large enough $\left(\varepsilon>\varepsilon_{c 2}\right)$, all agents can always form a single cluster, where $P_{c}$ equals to 1 . To be noted, the optimization of $P_{c}$ by $\alpha$ can be observed by careful scrutiny of $N_{c}$ shown in the inset of Figure 4(c). The curve of $N_{c}$ for $\alpha=0.1$ is first reaching 1 with the increase of $\varepsilon$, which implies the existence of the optimal $\alpha$ for obtaining the consensus state.

To further show the effects of different $\alpha$ on the opinion formation, we present the distribution of opinions $P\left(x_{i}\right)$ in the steady states in Figure 5, which is based on all different 


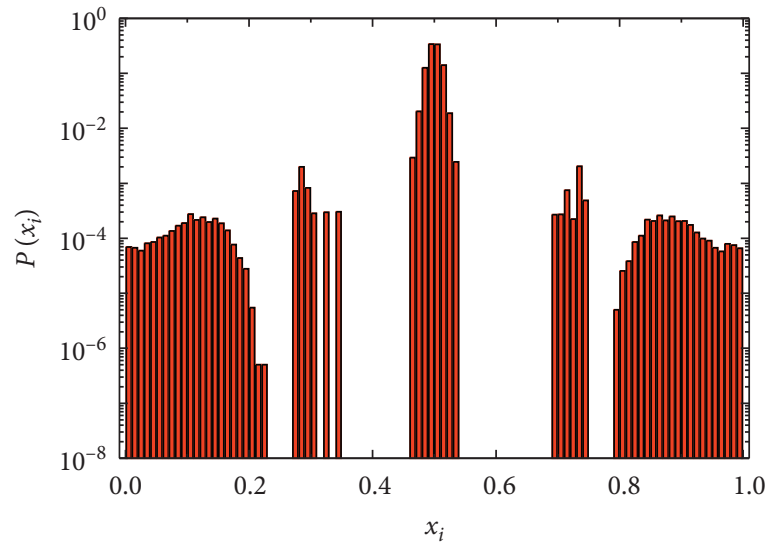

$\alpha=0$

(a)

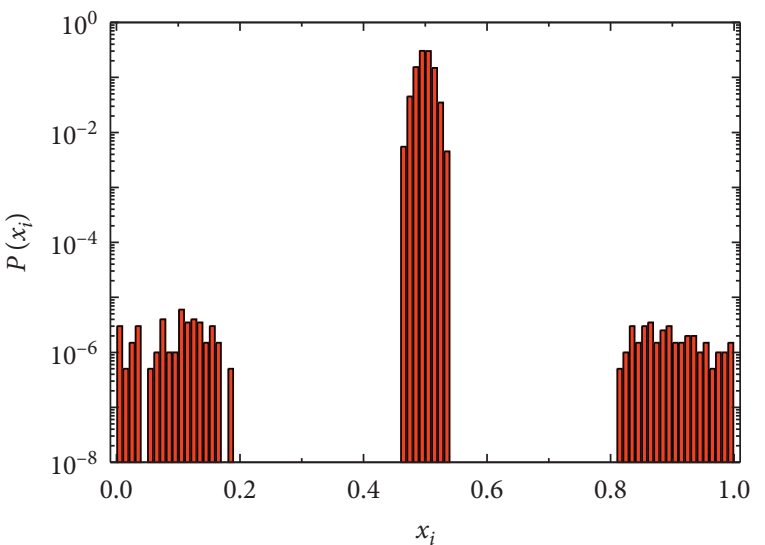

$\alpha=0.5$

(c)

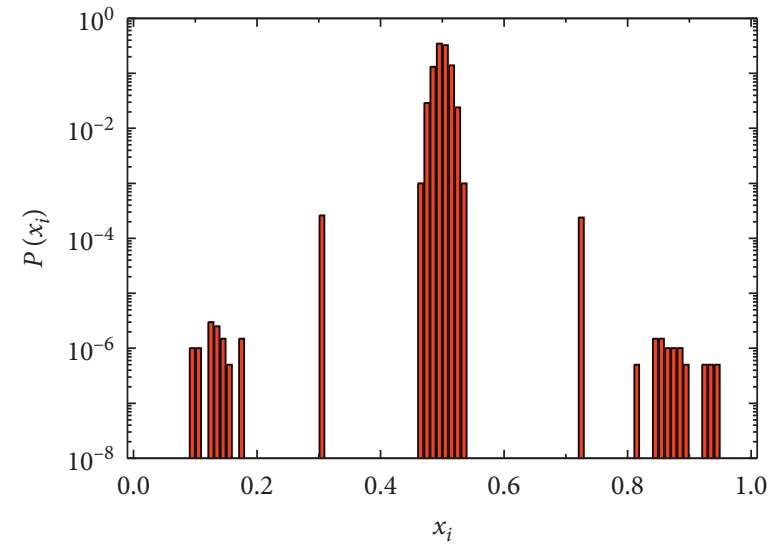

$\alpha=0.1$

(b)

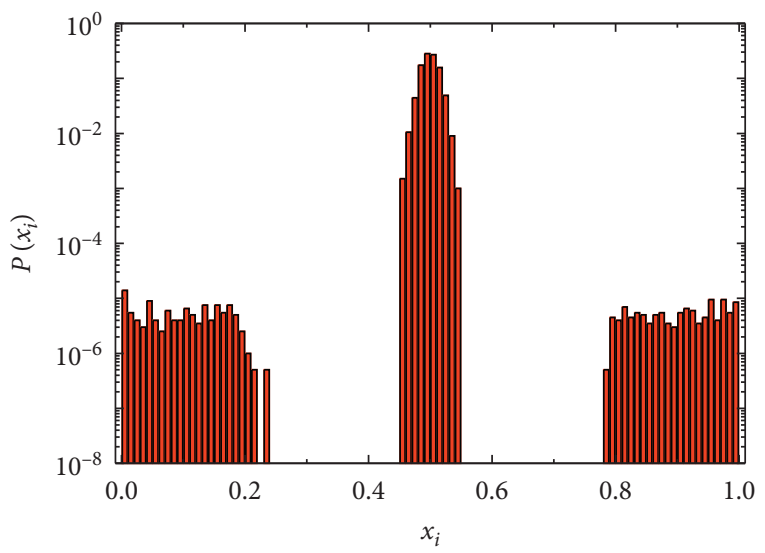

$\alpha=1$

(d)

Figure 5: The distribution of opinions in the population $P\left(x_{i}\right)$ at $\alpha=0,0.1,0.5$, and $1 . \varepsilon=0.3$.

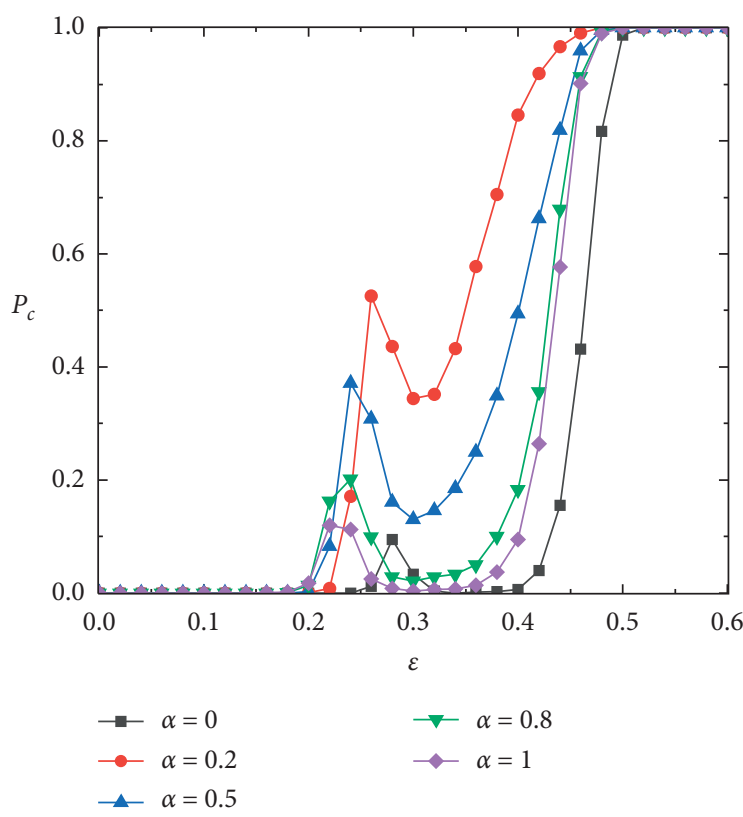

(a)

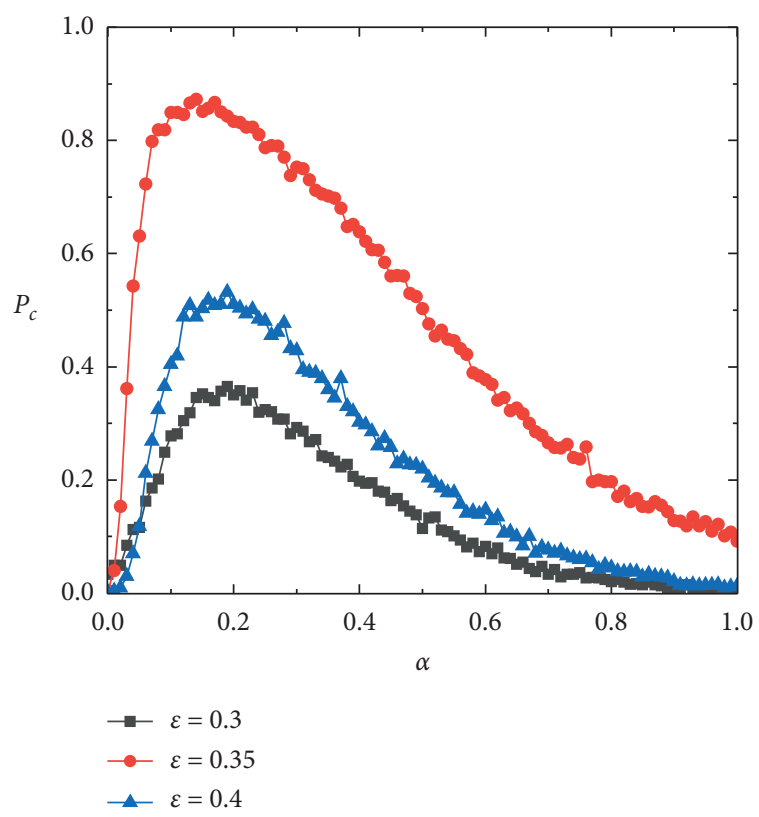

(b)

FIGURE 6: The results for the model on ER random networks (the average degree $\langle k\rangle=20$ ). (a) The probability of complete consensus $P_{c}$ as a function of $\varepsilon$ at $\alpha=0,0.2,0.5,0.8$, and 1 . (b) The probability of complete consensus $P_{c}$ as a function of $\alpha$ at $\varepsilon=0.3,0.35$, and 0.4 . 
realizations. From Figure 5, it can be easily found that $P\left(x_{i}\right)$ is symmetrical about $x_{i}=0.5$. When $\alpha$ is close to the optimal value which maximizes $P_{c}$ (for example, $\alpha=0.1$ ), the agents' opinions in the population are mainly concentrated around 0.5 . In contrast, for other $\alpha$, the distributions become fragmented. It is worth noting that $\alpha=0$ and 1 correspond to a single type of interaction, pairwise or group one, respectively. By comparison, we can conclude that the combination of these two types of interactions could efficiently promote consensus in the population.

Finally, we investigate the model on Erdös and Rényi (ER) random networks [31] instead of a complete graph, and the results are shown in Figure 6. One can see that the results for ER networks remain qualitatively unchanged when compared with those obtained on a complete graph. From Figure 6(a), we can still find two critical points of $\varepsilon$. Once $\varepsilon$ goes beyond $\varepsilon_{c 1}$, the consensus state is possible to be reached and $P_{c}>0$, and when it exceeds $\varepsilon_{c 2}$, consensus can be always obtained and $P_{c}=1$. Moreover, $P_{c}$ first increases and then decreases with the increase of $\alpha$, and the optimization of $P_{c}$ by $\alpha$ could be easily found in Figure 6(b). The promotion effects of consensus by the combination of pairwise and group interactions are robust to the changes of the network topology structure.

\section{Conclusion}

In this work, we propose a novel opinion dynamics by combining pairwise interaction and group-based interaction in the model, which are based on the traditional DW model and HK model, respectively. In particular, we introduce a tunable parameter $\alpha$ to control the weights of these two types of interactions. By numerical simulating the model, we find that the combination of the two types of interactions could efficiently promote consensus in the population, and there exists an optimal $\alpha$ which can lead the consensus probability $P_{c}$ to its maximum. Moreover, we show that, at the optimal $\alpha$, a minimum critical bounded confidence $\varepsilon_{c 2}$ can be obtained, over which the system can always reach consensus. Furthermore, we present the results for the relative sizes of the largest and the second largest opinion clusters, the number of opinion clusters, and the distributions of opinions in the population to show the opinion formation in the steady states. We also check the robustness of the results on ER random networks.

For simplicity, we use the homogeneous bounded confidence in this study. However, it can be noticed that the heterogeneous bounded confidence is common in real life $[32,33]$. The opinion dynamics with heterogeneous bounded confidence can be considered in our model in the further research. Moreover, in this work, we assign a uniform $\alpha$ to the whole population. However, in the real society, the individuals have different roles. For example, some individuals are leaders while some are followers, and their opinions might be affected in different ways. Along this line, a potential extension to this work would be to consider leaders and followers have different weights for the two types of interactions through different social channels. That is, the values of $\alpha$ are related to the roles of individuals. The effects of such role-dependent $\alpha$ on consensus is also worthy of investigation in the future study.

\section{Data Availability}

The data used to support the findings of this study are available from the corresponding author upon request.

\section{Conflicts of Interest}

The authors declare that there are no conflicts of interest.

\section{Acknowledgments}

This work was supported by the National Natural Science Foundation of China (Grant nos. 11805021 and 12005043).

\section{References}

[1] C. Castellano, S. Fortunato, and V. Loreto, "Statistical physics of social dynamics," Reviews of Modern Physics, vol. 81, no. 2, pp. 591-646, 2009.

[2] D. Acemoglu and A. Ozdaglar, "Opinion dynamics and learning in social networks," Dynamic Games and Applications, vol. 1, no. 1, pp. 3-49, 2011.

[3] V. Sood and S. Redner, "Voter model on heterogeneous graphs," Physical Review Letters, vol. 94, no. 17, Article ID 178701, 2005.

[4] N. Masuda, "Voter models with contrarian agents," Physical Review E, vol. 88, no. 5, Article ID 52803, 2013.

[5] S. Galam, "Minority opinion spreading in random geometry," The European Physical Journal B, vol. 25, no. 4, pp. 403-406, 2002.

[6] R. Durrett, J. P. Gleeson, A. L. Lloyd et al., "Graph fission in an evolving voter model," in Proceedings of the National Academy of Sciences, vol. 109, no. 10, pp. 3682-3687, 2012.

[7] R. A. Holley and T. M. Liggett, "Ergodic theorems for weakly interacting infinite systems and the voter model," The Annals of Probability, vol. 3, no. 4, pp. 643-663, 1975.

[8] L. Dall'Asta, A. Baronchelli, A. Barrat, and V. Loreto, "Nonequilibrium dynamics of language games on complex networks," Physical Review E, vol. 74, no. 3, Article ID 36105, 2006.

[9] K. Sznajd-Weron and J. Sznajd, "Opinion evolution in closed community," International Journal of Modern Physics C, vol. 11, no. 6, pp. 1157-1165, 2000.

[10] A. Nowak, J. Szamrej, and B. Latané, "From private attitude to public opinion: a dynamic theory of social impact," Psychological Review, vol. 97, no. 3, pp. 362-376, 1990.

[11] G. Deffuant, D. Neau, F. Amblard, and G. Weisbuch, "Mixing beliefs among interacting agents," Advances in Complex Systems, vol. 3, pp. 87-98, 2000.

[12] R. Hegselmann and U. Krause, "Opinion dynamics and bounded confidence: models, analysis, and simulation," The Journal of Artificial Societies and Social Simulation, vol. 5, no. 3, 2002.

[13] S. Fortunato, "Universality of the threshold for complete consensus for the opinion dynamics of deffuant," International Journal of Modern Physics C, vol. 15, no. 9, pp. 13011307, 2004.

[14] C. Huang, Q. Dai, W. Han, Y. Feng, H. Cheng, and H. Li, "Effects of heterogeneous convergence rate on consensus in opinion dynamics," Physica A: Statistical Mechanics and Its Applications, vol. 499, pp. 428-435, 2018.

[15] Y. Shang, "Deffuant model of opinion formation in onedimensional multiplex networks," Journal of Physics A: Mathematical and Theoretical, vol. 48, no. 39, Article ID 395101, 2015. 
[16] G. Chen, H. Cheng, C. Huang et al., "Deffuant model on a ring with repelling mechanism and circular opinions," Physical Review E, vol. 95, no. 4, Article ID 42118, 2017.

[17] J. Huang and X. Jin, "Opinion formation on evolving and adaptive networks," Journal of Statistical Mechanics: Theory and Experiment, vol. 2019, Article ID 13202, 2019.

[18] H. Liang, Y. Yang, and X. Wang, "Opinion dynamics in networks with heterogeneous confidence and influence," Physica A: Statistical Mechanics and Its Applications, vol. 392, no. 9, pp. 2248-2256, 2013.

[19] J. Lorenz, "Heterogeneous bounds of confidence: meet, discuss and find consensus!," Complexity, vol. 15, no. 4, pp. 43-52, 2009.

[20] G. Fu, W. Zhang, and Z. Li, "Opinion dynamics of modified Hegselmann-Krause model in a group-based population with heterogeneous bounded confidence," Physica A: Statistical Mechanics and Its Applications, vol. 419, pp. 558-565, 2015.

[21] G. Kou, Y. Zhao, Y. Peng, and Y. Shi, "Multi-level opinion dynamics under bounded confidence," PLoS One, vol. 7, no. 9, Article ID e43507, 2012.

[22] Y. Shang, "Consensus formation of two-level opinion dynamics," Acta Mathematica Scientia, vol. 34, no. 4, pp. 1029-1040, 2014.

[23] X. Chen, X. Xiong, M. Zhang, and W. Li, "Public authority control strategy for opinion evolution in social networks," Chaos, vol. 26, no. 8, Article ID 83105, 2016.

[24] W. Han, C. Huang, and J. Yang, "Opinion clusters in a modified hegselmann-krause model with heterogeneous bounded confidences and stubbornness," Physica A: Statistical Mechanics and Its Applications, vol. 531, Article ID 121791, 2019.

[25] J. Ghaderi and R. Srikant, "Opinion dynamics in social networks with stubborn agents: equilibrium and convergence rate," Automatica, vol. 50, no. 12, pp. 3209-3215, 2014.

[26] A. Olshevsky and J. N. Tsitsiklis, "Convergence speed in distributed consensus and averaging," Siam Review, vol. 53, no. 4, pp. 747-772, 2011.

[27] X. Chen, Z. Wu, H. Wang, and W. Li, "Impact of heterogeneity on opinion dynamics: heterogeneous interaction model," Complexity, vol. 2017, Article ID 5802182, 10 pages, 2017.

[28] N. L. Abrica-Jacinto, E. Kurmyshev, and H. A. Juárez, "Effects of the interaction between ideological affinity and psychological reaction of agents on the opinion dynamics in a relative agreement model," The Journal of Artificial Societies and Social Simulation, vol. 20, no. 3, 2017.

[29] L. Shang and S. Chen, "Opinion dynamics with decentralized proportional-integral control strategy," Physica A: Statistical Mechanics and Its Applications, vol. 533, Article ID 121916, 2019.

[30] X. Chen, S. Zhao, and W. Li, "Opinion dynamics model based on cognitive styles: field-dependence and field-independence," Complexity, vol. 2019, Article ID 2864124, 12 pages, 2019.

[31] P. Erdös and A. Rényi, "On the evolution of random graphs," Mathematical Institute of the Hungarian Academy of Sciences, vol. 5, pp. 17-60, 1960.

[32] Y. Shang, "An agent based model for opinion dynamics with random confidence threshold," Communications in Nonlinear Science and Numerical Simulation, vol. 19, no. 10, pp. 37663777, 2014.

[33] M. Pineda and G. M. Buendía, "Mass media and heterogeneous bounds of confidence in continuous opinion dynamics," Physica A: Statistical Mechanics and Its Applications, vol. 420, pp. 73-84, 2015. 